\title{
Radiation Damage on Liquid Electrolyte during Spatially Resolved Soft X-ray Photoemission Measurements
}

\author{
Christopher Arble, ${ }^{1 *}$ Hongxuan Guo ${ }^{2}$, Brian Hoskins, ${ }^{1}$ Matteo Amati, ${ }^{3}$ Patrick Zeller, ${ }^{3}$ Luca \\ Gregoratti, ${ }^{3}$ and Andrei Kolmakov ${ }^{1 *}$ \\ 1. Center for Nanoscale Science and Technology, National Institute of Standards and \\ Technology, Gaithersburg, Maryland 20899, United States \\ 2. SEU-FEI Nano-Pico Center, Key Laboratory of MEMS of Ministry of Education, Southeast \\ University, Nanjing 210096, P. R. China \\ 3. Elettra Sincrotrone Trieste SCpA, SS14, Km163-5, I-34149 Trieste, Italy \\ * Corresponding author: christopher.arble@nist.gov and andrei.kolmakov@nist.gov
}

Chemical processes occurring at solid-liquid interfaces play a key role in many important industrial and biomedical applications, including energy harvesting, storage, conversion, heterogeneous catalysis, medical implants, etc. [1,2]. X-ray photoelectron spectroscopy (XPS) is ideally suited to probe elemental and chemical information of surfaces and interfaces with nanoscale spatial sensitivity but has traditionally been restricted to ultra-high vacuum (UHV) environments due to the prohibitively large scattering cross-section of outgoing electrons within condensed and gaseous media. Over the past few years, advances in applications of two dimensional (2D) materials, i.e. graphene, resulted in the implementation of electron-based microscopy and spectroscopy to probe liquid interfaces by employing 2D materials as molecularly impermeable, electron transparent membranes to separate the sample environment from UHV conditions in the analysis chamber [35]. Within this study, graphene liquid cells were used to probe electrochemistry of a model $\mathrm{CuSO}_{4}$ system with XPS in-operando mode using scanning photoelectron microscopy (SPEM) while the effects due radiolysis of the solution imparted from the synchrotron photon beam were monitored in morphology and speciation. These results were complemented with scanning electron microscopy (SEM) and energy dispersive X-ray spectroscopy (EDS) measurements.

A liquid cell sample platform was made as an array of electrolyte filled several thousand isolated micro-channels, with a diameter of 2 to $5 \mu \mathrm{m}$ and encapsulated with bilayer graphene (BLG) on the front side and with water-immiscible epoxy on the backside(Fig. 1A)[6]. Highly focused Xray beam was rastered over the graphene covered channel to elucidate chemical composition of the electrode-electrolyte interface and its evolution during metal plating/stripping reactions. We found that highly focused X-ray radiation causes radiolysis of the electrolyte which may dominate the system behavior during the electrochemical experiment (Fig. 1C). Deconvolution of the spectra identified oxidized species of copper and oxygen as well as reduced states of sulfur that were interrelated to the X-ray exposure of the sample. Corresponding SEM images taken after focusing the electron beam onto the central region of the graphene liquid cell window and subsequently collecting EDS spectral maps display spatial confided irradiated byproducts which can be associated with the species observed in with XPS (Fig. 2).

In both photoelectron and electron-based microscopies employed on liquid cells in this study revealed radiation-induced byproducts that became preferentially bound to the graphene membrane. The influence of beam effects on liquid samples has been already reported during liquid jet XPS studies [7]. In our case, XPS observations of the graphene electrode-liquid 
electrolyte interface showed that under the X-ray flux ca $10^{12} \mathrm{ph} / \mathrm{sec}$ typical for SPEM imaging, the electrochemical deposition of $\mathrm{Cu}$ was overshadowed by the effects of radiolysis of the solution. The sensitivity of XPS towards the chemical composition of the electrode/electrolyte interface and radiolitic products suggests that the membrane approach is feasible if the parametric space of the experiment can mitigate radiolysis threshold of the solution, i.e. lower dose, microfluidics, etc.

\section{References:}

1. J, Saveant, Chemical Reviews 108 (2008), p. 2348.

2. D. Larcher, J. Tarascon, Nature Chemistry 7 (2015), p. 19.

3. A. Kolmakov et al, Nature Nanotechnology 6 (2011) p. 651.

4. J.J. Velasco-Velez et al, Angewandte Chemie International Edition 54 (2015). p. 14554.

5. R. Weatherup, Topics in Catalysis 61 (2018). p. 2085.

6. A. Yulaev et al, ACS Applied Materials and Interfaces, 9 (2017) p. 26492.

7. R. Golnak et al, Scientific Reports 6 (2016) p. 1.
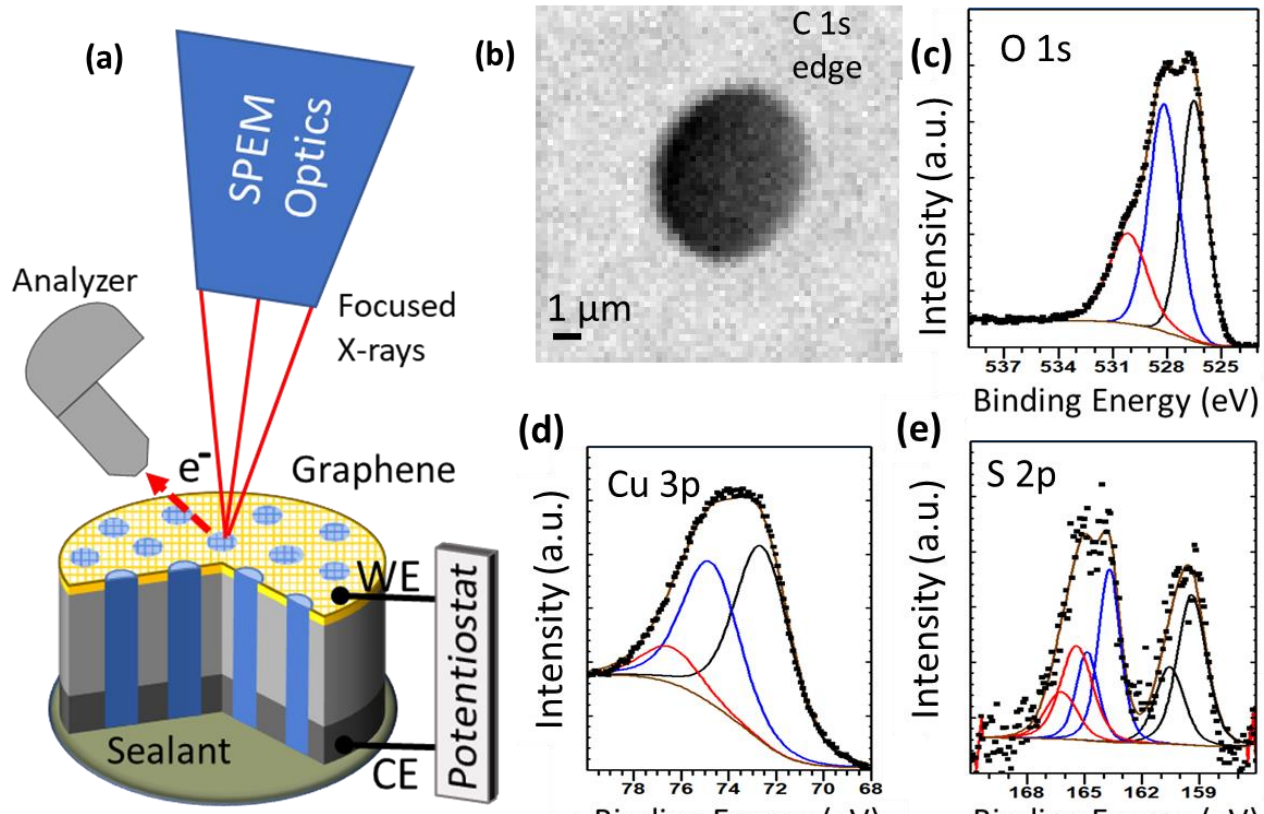

Figure 1. a) Liquid cell and experimental setup. b) SPEM C 1s mapping of liquid cell. c, d, e) XPS spectra and fittings of $\mathrm{O} 1 \mathrm{~s}, \mathrm{Cu} 3 \mathrm{p}$, and $\mathrm{S} 2 \mathrm{p}$ respectively.

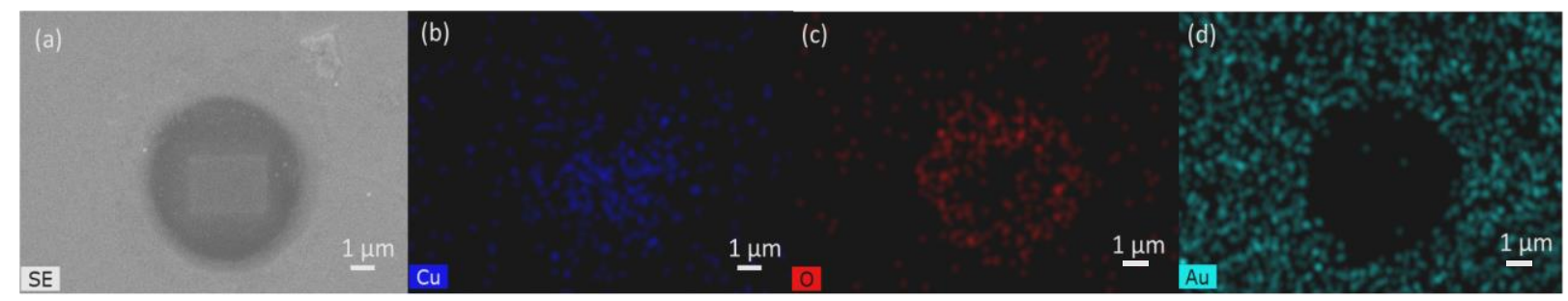

Figure 2. a) SEM image of the individual channel of graphene liquid cell after intentional extensive exposure of the central part with $8 \mathrm{keV}$ electrons. b, c, d) corresponding EDS elemental maps of $\mathrm{Cu}, \mathrm{O}$ and $\mathrm{Au}$. 